\title{
Exploring the translational disconnect between the murine and human inflammatory response: analysis of LPS dose-response relationship in murine versus human cell lines and implications for translation into murine models of sepsis
}

This article was published in the following Dove Press journal:

Journal of Inflammation Research

16 October 2015

Number of times this article has been viewed

\section{Eamon P McCarron' \\ Dominic P Williams' \\ Daniel J Antoine' \\ Anja Kipar ${ }^{2}$ \\ Jana Lemm ${ }^{3}$ \\ Sebastian Stehr ${ }^{3}$ \\ Ingeborg D Welters ${ }^{4}$}

'Department of Clinical and Molecular Pharmacology, Centre for Drug Safety Science, Institute of Translational Medicine, ${ }^{2}$ Department of Veterinary Pathology, University of Liverpool, Liverpool, UK; ${ }^{3}$ Department of Anaesthesiology and Intensive Care Medicine, Jena University Hospital, Jena, Germany; ${ }^{4}$ Department of Obesity and Endocrinology, Institute of Ageing and Chronic Disease, University of Liverpool, Liverpool, UK

Correspondence: Eamon P McCarron Department of Clinical and Molecular Pharmacology, Centre for Drug Safety Science, Institute of Translational Medicine, University of Liverpool, Ashton Street, Liverpool, L69 3GE, UK Email e.p.mccarron@doctors.org.uk
Background: Inflammation forms an important part of the human innate immune system and is largely dependent on the activation of the "classical" NF- $\kappa B$ pathway through Toll-like receptors (TLRs). Understanding this has allowed researchers to explore roles of therapeutic targets in managing conditions such as sepsis. Recapitulating an inflammatory response using lipopolysaccharide (LPS), a "sterile" technique, can provide information that is dissimilar to the clinical condition. By examining NF- $\kappa \mathrm{B}$ activation (through immunoblotting of the p65 subunit) in two separate cell lines (murine and human) and analyzing two murine models of sepsis (intraperitoneal [IP] LPS and IP stool inoculation), an evaluation of the translational disconnect between experimental and clinical sepsis can be made.

Methods: THP-1 (human) cells and RAW 264.7 (murine) cells were dosed with concentrations of LPS (human, $1 \mathrm{pg} / \mathrm{mL}$ to $100 \mathrm{ng} / \mathrm{mL}$; murine, $30 \mathrm{pg} / \mathrm{mL}$ to $1,000 \mathrm{ng} / \mathrm{mL}$ ) and nuclear actin and p65 were immunoblotted to measure changes in nuclear density. In vivo, C57BL/6 mice received either IP injection of stool suspension $(5 \mu \mathrm{L} / \mathrm{g})$ or LPS $(25 \mathrm{mg} / \mathrm{kg})$ or saline $(1 \mathrm{~mL} / \mathrm{kg})$. Animals were culled at 6 hours and tissues were analyzed.

Results: An increase in basal p65:actin density in THP-1 cells (mean 0.214, standard error of the mean 0.024$)$ was seen at doses as small as $0.1 \mathrm{ng} / \mathrm{mL}(0.519 \pm 0.064)$. In contrast to RAW 264.7 cells, basal increases $(0.170 \pm 0.025)$ were only seen when a dose of $3 \mathrm{ng} / \mathrm{mL}(0.387 \pm 0.078)$ was used. Dose-response analysis of p65:actin ratio showed that THP-1 cells respond to lower doses of LPS than RAW 264.7 cells and lower doses produce a greater fold increase in the nuclear p65 density. Both in vivo models showed evidence of neutrophil (NL) recruitment into tissues (which was more intense after LPS treatment). IP stool inoculation resulted in an acute suppurative peritonitis and more substantial evidence of NL recruitment into adipose tissue and skeletal muscle.

Conclusion: Our results support previous observations that translation of murine models into the human clinical setting suffers from considerable limitations including species-associated differences in LPS response seen at a molecular level. Furthermore, the histopathological changes during clinical sepsis cannot be adequately reproduced by injection of LPS. Therefore, the so-called translational disconnect that exists between murine LPS models and human sepsis involves NF- $\mathrm{\kappa B}$ activation at a molecular level and is further augmented by the use of LPS as a stimulus for infectious responses in vivo.

Keywords: NF-кB, p65, LPS, murine, sepsis, inflammation, leukocyte, monocyte, macrophage 


\section{Background}

Inflammation is dependent on cytokine production, which, in humans (and mice), is largely a result of "classical" $\mathrm{NF}-\mathrm{KB}$ activation. This is mediated through the action of pathogen-associated molecular patterns on Toll-like receptors (TLRs) ${ }^{1,2}$ Lipopolysaccharide (LPS) is a prototypical pathogen-associated molecular pattern and potent activator of TLRs. Activation of TLRs by LPS (namely TLR4) allows $\mathrm{NF}-\mathrm{\kappa B}$ to translocate from the cytoplasm to the nucleus, inducing target gene transcription through the nuclear localization sequence (NLS). ${ }^{1}$ This leads to the translocation of the p50-p65 heterodimer and increased transcription of genes coding for cytokines (ie, tumor necrosis factor alpha $[\mathrm{TNF} \alpha])$ and subsequent inflammation. ${ }^{3}$

Sepsis is a life-threatening clinical condition defined by the American College of Chest Physicians/Society of Critical Care Medicine (ACCP/SCCM) as a systemic inflammatory response syndrome together with a demonstrable source of infection. ${ }^{4}$ LPS, a bacterial endotoxin from Gram-negative bacteria, plays a major role in eliciting this inflammatory response in Gram-negative sepsis. Hence, LPS is widely used in animal models of sepsis. ${ }^{5}$ In experimental animals, a bolus injection of intraperitoneal (IP) LPS will induce a sharp, but a transient increase in pro-inflammatory cytokines such as TNF $\alpha$, IL-1, and IL-6, whereas in human sepsis, the host response is triggered by live bacteria, giving detectable cytokine levels that are significantly lower, but sustained over a longer period of time. ${ }^{6}$ Despite the disparities, LPS is widely used in research because models are relatively straightforward, produce the greatest homogeneity among all in vivo models of sepsis, and are, thus, relatively easy to replicate. ${ }^{7}$

Doi et $\mathrm{al}^{8}$ stated in their review on animal models of sepsis that "murine sepsis should mimic the pace and severity of human sepsis; reproduce key hemodynamic and immunological stages and mimic histology findings in the key organs lung, liver, spleen, kidney". Insufficiencies in experimental models of sepsis are accepted causes for the lack of efficacy of developed therapeutic agents in humans. This is most notable in the case of the development of monoclonal antibodies against TNF $\alpha$, which improved survival in LPS-treated mice, but failed to have a similar translational effect in humans. ${ }^{9}$

Gonnert et $\mathrm{al}^{10}$ developed an IP stool inoculation model in mice that was able to induce clinically significant, quantifiable, and reproducible symptoms of sepsis in rodents. The stool was inoculated into the peritoneum of mice and induced a cytokine response similar to kinetics of IL-6 and
IL-10 observed in human sepsis. Microbiological analysis showed that the stool contained Escherichia coli and Bacteroides species, important Gram-negative organisms that are known causatives of sepsis in humans. ${ }^{11}$

Recapitulating an inflammatory response using "sterile" techniques, such as LPS injection, can provide information that is dissimilar to human sepsis. By examining this in two separate cell lines (murine and human), we aim to compare differences between species. Comparing two cell populations can also provide information about the role of two separate immune cells in inflammation, their respective sensitivities to LPS and their capacity for cytokine production. In addition, we aim to demonstrate parallels and differences in the time course and magnitude of the cytokine release by comparing the new inoculation model with classical LPS challenge. This will help to identify a mice model that is most representative of human sepsis.

\section{Materials and methods Materials}

THP-1 human leukemic monocytes (catalog number 88081201, Sigma-Aldrich Co, St Louis, MO, USA) were cultured in RPMI-1640 (catalog number R8758, Sigma-Aldrich Co). RAW 264.7 murine macrophages (catalog number 91062702, Sigma-Aldrich Co) were cultured in Dulbecco's Modified Eagle's Medium (DMEM, Lonza, Basel, Switzerland). LPS used in dosing was obtained by phenol extraction from E. coli 0111:B4 (catalog number L2630, Sigma-Aldrich Co). Mouse monoclonal IgG against p65 (Santa Cruz Biotechnology, Inc. (catalog number sc-109, Dallas, TX, USA); mouse monoclonal IgG against $\beta$-actin (Abcam, Cambridge, UK, catalog number mAbcam 8224), Goat-derived monoclonal anti-mouse IgG (catalog number A0168, Sigma-Aldrich Co). Samples from the IP stool inoculation model were collected from eleven C57BL/6 mice at necropsy and included liver, gastrocnemius muscle, epididymal, peri-renal, and subcutaneous fat. Samples were fixed in $4 \%$ paraformaldehyde and embedded in paraffin cassettes before being shipped to the UK (Department of Anaesthesiology and Intensive Care Medicine, Jena University Hospital, Jena, Germany). Mice used in the IP LPS dosing experiment were 22 male C57BL $/ 6$ mice (25-30 g) obtained from Charles River (Margate, UK). LPS used in dosing was obtained by phenol extraction from E. coli 0111:B4 (catalog number L2630, Sigma-Aldrich Co). Sodium chloride for saline (catalog numbers A7085 and S7653, Sigma-Aldrich Co) along with LPS were all in powder form and were reconstituted in distilled water before administration. 


\section{Cell culture and dosing}

All cell culture work was carried out in a sterilized cell culture hood using an aseptic technique. THP-1 cells were cultured in RPMI-1640 cell culture medium treated with $10 \%$ fetal bovine serum, L-glutamine, and streptomycin. Cells were seeded at $5 \times 10^{6}$ per $\mathrm{mL}$ in $7 \mathrm{~mL}$ of media within a smaller T 25 cell culture flask and incubated overnight before dosing. Eight flasks in total $\left(40 \times 10^{6}\right.$ cells $)$ were required for the experiment - seven doses and a negative control. Cells were only used for experiment during passage 9-11. Cells were dosed with LPS in seven doses $(0.1,0.3,1,3,10,30$, and $100 \mathrm{ng} / \mathrm{mL}$ ).

RAW 264.7 cells were cultured in DMEM treated with $10 \%$ fetal bovine serum, L-glutamine, and streptomycin. For the experiment, cells were seeded at $1 \times 10^{6}$ cells per $\mathrm{mL}$ in $5 \mathrm{~mL}$ in a T25 flask and incubated overnight before dosing. Seven flasks in total $\left(35 \times 10^{6}\right.$ cells $)$ were required for the experiment - six doses and a negative control. Cells were only used for experiment during passage 15-20. Cells were dosed with LPS in six doses $(3,10,30,100,300$, and $1,000 \mathrm{ng} / \mathrm{mL})$. Optimization demonstrated that significant elevations in basal p65 in RAW cells, when stimulated with LPS, were only seen at concentrations of $3 \mathrm{ng} / \mathrm{mL}$ and above. Similar numbers of cells were used in each experiment (THP- 1 cells: $5 \times 10^{6}$ in $7 \mathrm{~mL}$ of media, RAW cells: $5 \times 10^{6}$ in $5 \mathrm{~mL}$ of media).

\section{Electrophoresis and immunoblotting}

The cell nuclear fractions were isolated and loaded onto acrylamide gels. Gels were cast using gel casting equipment and reagents obtained from Bio-Rad Laboratories Inc., (Hercules, CA, USA). In all, 7.5\% acrylamide gels were used. Five micrograms of each nuclear extract was loaded onto the gel with a standard protein ladder (Bio-Rad Kaleidoscope). In brief, $90 \mathrm{~V}$ was applied for 10 minutes then increased to $150 \mathrm{~V}$ for 45 minutes. The proteins were then transferred onto a nitrocellulose membrane in a transfer buffer (buffer $=100 \mathrm{~mL} 10 \times$ buffer containing glycine and Tris base [Bio-Rad], $200 \mathrm{~mL}$ ethanol and $700 \mathrm{~mL}$ of distilled water).
A current of 80 volts (V) was supplied for 1 hour and sufficient transfer was detected using ponceau red stain. Membranes were washed with $0.1 \% / 1 \times$ tris-buffered saline (TTBS) and blocked for 45 minutes while being agitated in 10\% analytical grade nonfat milk (Bio-Rad). The membranes were then cut, based on the respective molecular weights; the $>50 \mathrm{kDa}$ section was incubated primarily with mouse monoclonal anti-p65 antibody (Santa Cruz Biotechnology) made up in $2 \%$ nonfat milk to a concentration of 1:1,000, while the lower section was incubated primarily with mouse monoclonal anti$\beta$-actin (AbCam) made up to a dilution of $1: 160,000$ in $2 \%$ nonfat milk. Both were incubated for 1 hour while constantly being agitated. Membranes were then washed four times for 5 minutes with $0.1 \% / 1 \times$ TTBS. Both membranes were then incubated with goat anti-mouse IgG (Sigma-Aldrich Co) as the secondary antibody. The membrane for p65 detection was incubated at a concentration of 1:5,000 made up in $2 \%$ milk, while the $\beta$-actin membrane was exposed to a secondary antibody dilution of 1:10,000. Chemiluminescence (ProteoQwest ${ }^{\mathrm{TM}}$ ) was used to develop the films and membranes were exposed to films for 1 minute to detect p65 and 10 seconds to detect actin (Figure 1).

\section{Densitometry and statistical analysis}

Films were developed and scanned onto a computer and using subtraction analysis on VisionWorks ${ }^{\circledR}$, values for densities of p65 and actin bands were calculated. This allowed a p65:actin ratio to be calculated where increases in the density of p65 corresponded to increasing values. Data were interpreted on Microsoft Excel ${ }^{\circledR}$ and curves were constructed using GraphPad Prism $5^{\circledR}$. Three repeats were carried out for each cell line $(\mathrm{N}=3)$; this allowed means, standard deviation, and standard error of the mean to be calculated.

\section{Animal experiments}

In the UK experiments 22 male $\mathrm{C} 57 / \mathrm{BL} / 6$ mice male $\mathrm{C} 57 /$ $\mathrm{BL} / 6$ mice $(25-30 \mathrm{~g})$ were allowed to acclimatize for 1 week prior to the experiment. Animals were treated in accordance

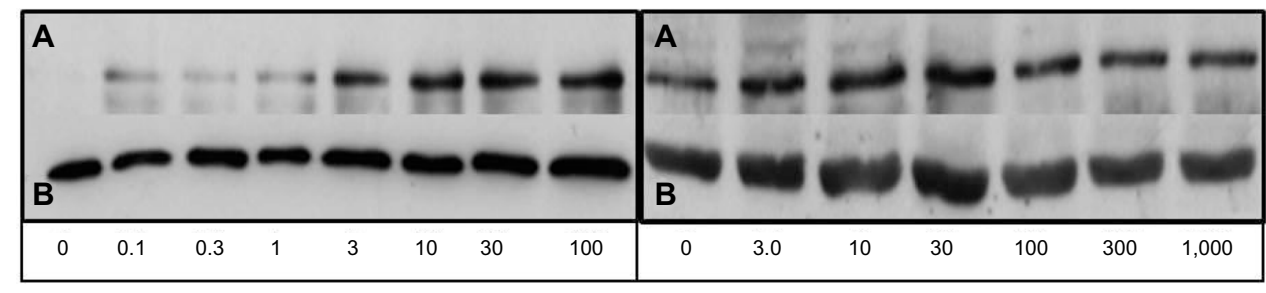

Figure I Films of immunoblots for nuclear p65 in THP-I cells (left) and RAW 264.7 cells (right) dosed with LPS after I hour.

Notes: Films are displayed with corresponding LPS dose below in $\mathrm{ng} / \mathrm{mL}$. On top are $\mathrm{p} 65 \mathrm{I}$-minute films (A) along with 10 -second actin films on the bottom (B). This figure is representative of three experiments $(\mathrm{N}=3)$.

Abbreviation: LPS, lipopolysaccharide. 
with guidelines set out by the Animals (Scientific Procedures) Act 1986 (ASPA) (project licence 40/3143) with regard to the handling and experimentation of animals. Throughout the experiment, animals had free access to food and water and were kept in cages. Similarly, in German experiments all animal procedures were performed in strict compliance with the German legislation regarding protection of animals. All experiments were approved by the regional animal welfare committee (Thuringian State Office for Food Safety and Consumer Protection, Department for Animal Welfare, Bad Langensalza, Thuringia, Germany) and all efforts were made to minimize suffering.

\section{Dosing regimen of C57BL/6 mice with LPS, stool suspension, and saline}

Animals were weighed, marked, and divided into two groups and given one of the following treatments IP: $25 \mathrm{mg} / \mathrm{kg}$ LPS (a dose known to induce lethal endotoxemia) or normal saline (an equivalent amount to that used in LPS dosing) and culled using carbon dioxide suffocation at 6 hours to allow comparison with stool inoculation. All animals were given subcutaneous saline to compensate for fluid volume of $25 \mu \mathrm{L} / \mathrm{g}$ body weight. In a second series of experiments, male C57BL/6 mice (25-30 g) received an IP injection (right upper quadrant) for induction of sepsis. Referring to a recently published rodent model (Gonnert et $\mathrm{al}^{10}$ ), male C57BL/6 mice $(25-30 \mathrm{~g})$ were subjected to peritoneal contamination and infection (PCI). Animals received an intraperitoneal injection (left lower abdomen) of $5 \mu \mathrm{L} / \mathrm{g}$ body weight (BW) of a standardized 1:4 diluted (saline) human feces suspension (4.25 $\times 10^{7} \mathrm{CFU} / \mathrm{g}$ BW; predicted to be highly lethal). All animals were given subcutaneous saline to compensate for fluid volume of $25 \mu \mathrm{L} / \mathrm{g}$ BW. Six hours after sepsis induction, animals were euthanized in deep anesthesia with isoflurane, and tissue samples removed including the liver, epididymal, peri-nephritic, and subcutaneous fat. Untreated mice were used as controls.

\section{Tissue fixation and preparation}

Tissue was fixed in 4\% paraformaldehyde before being cut into an appropriate size and placed in a cassette and tissue processor overnight to remove water and replace it with paraffin wax. Within the processor, tissues are dehydrated using increasing concentrations of ethanol, and excess ethanol was removed using xylene. Finally, molten paraffin wax replaces xylene and the tissue is embedded into moulds before sectioning. Basic staining (hematoxylin and eosin) was carried out for a general histopathological assessment of the tissue. Periodic acid-Schiff (PAS) was also done where appropriate.

\section{Results \\ Detection of p65 translocation in the nuclear extract of THP-I cells, I hour after dosing with LPS}

Increasing the concentrations of LPS stimulated translocation of p65 from the cytoplasm to the nucleus (Figure 2), in a dose-response relationship (Figure 3). An increase in basal p65 density $(0.214 \pm 0.0238)$ was seen at doses as small as $0.1 \mathrm{ng} / \mathrm{mL}(0.519 \pm 0.0641)$. The coefficient of variation values for the experiments was between $11.5 \%$ and $27.6 \%$ for individual doses.

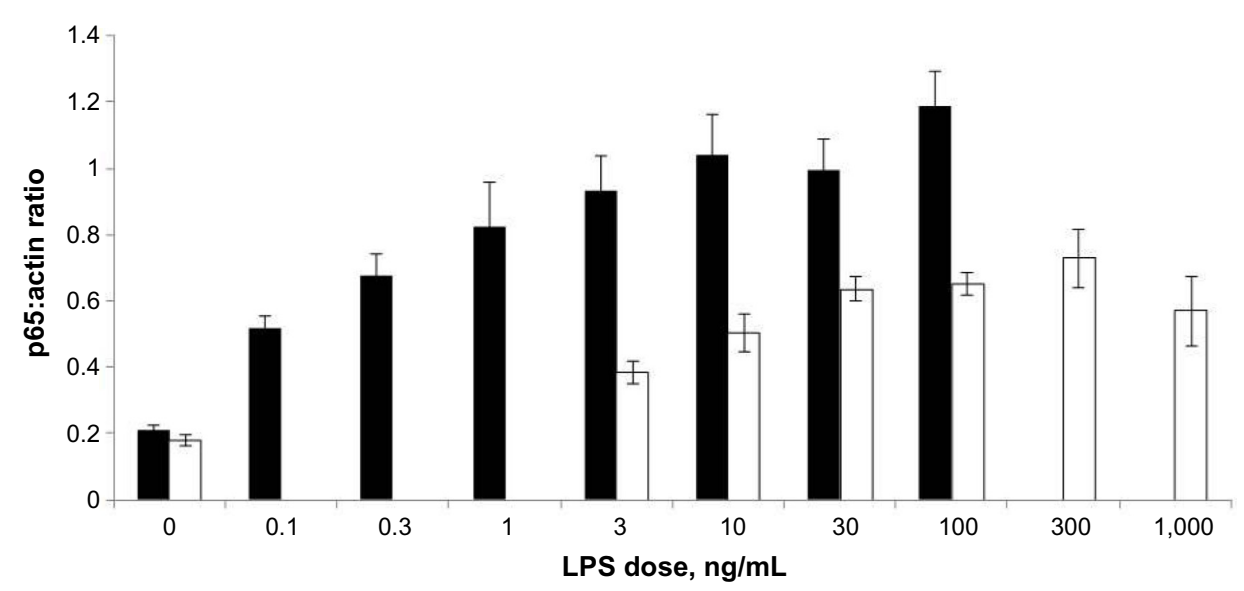

Figure 2 Results of densitometry on developed films of immunoblots of nuclear extract from THP-I cells (black) and RAW 264.7 (white) dosed with varying increasing concentrations of LPS $(\mathrm{N}=3)$.

Notes: Bar charts are displayed with P65:actin ratio mean on the $Y$-axis and LPS dose $(\mathrm{ng} / \mathrm{mL})$ on the $X$-axis. Means are plotted with standard error of the means. Experiments were repeated three times $(\mathrm{N}=3)$.

Abbreviation: LPS, lipopolysaccharide. 

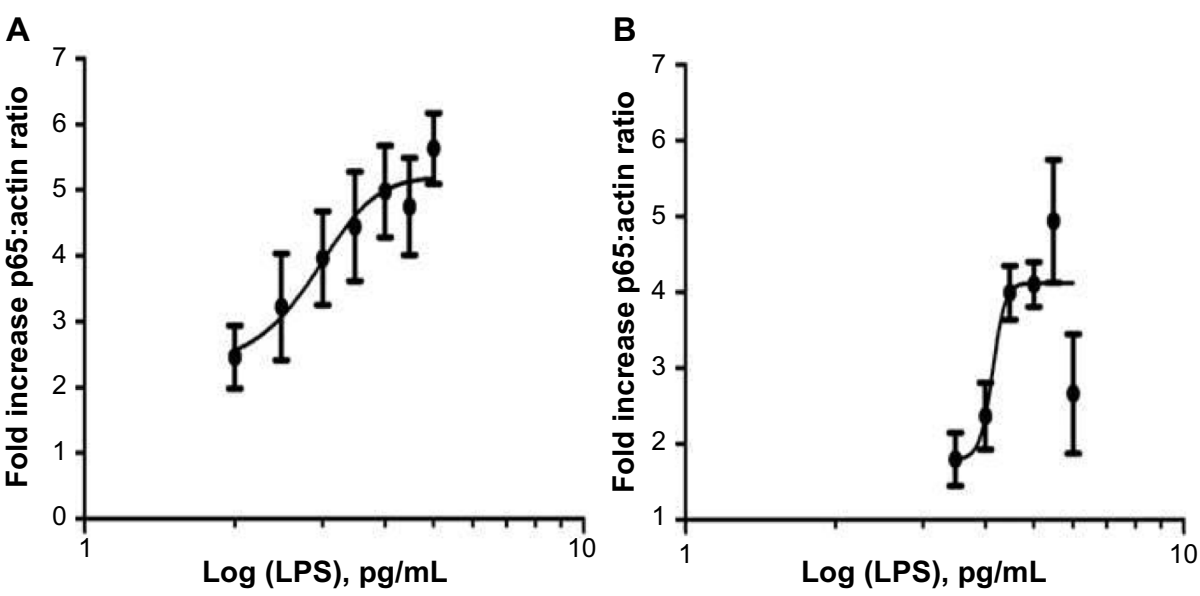

Figure 3 Results of dose response analysis for LPS dose vs p65:actin ratio in THP-I and RAW 264.7 cell lines.

Notes: Dose-response curves for (A) THP-I and (B) RAW 264.7 cell lines dosed with varying concentrations of LPS. The X-axis is the log values of the concentrations with fold increase in the $\mathrm{p} 65$ :actin ratio on the $Y$-axis. Fold increase was calculated by dividing the mean basal p65 response into all other mean doses. Means are plotted with SEM. Experiments were repeated three times $(\mathrm{N}=3)$.

Abbreviations: LPS, lipopolysaccharide; SEM, standard error of the mean.

\section{Detection of p65 translocation in the nuclear extract of RAW 264.7 cells, I hour after dosing with LPS}

Increasing the concentrations of LPS stimulated translocation of p65 from the cytoplasm to the nucleus (Figure 2), in a dose-response relationship (Figure 3). An increase in basal p65 density $(0.170 \pm 0.025)$ was only seen when a dose of $3 \mathrm{ng}$ / $\mathrm{mL}(0.387 \pm 0.078)$ was used (which is much higher in comparison to $0.1 \mathrm{ng} / \mathrm{mL}$ used for THP-1 cells). In this particular cell line, much higher concentrations of LPS were required in order to stimulate the cells, and a maximal response was seen between 100 and $300 \mathrm{ng} / \mathrm{mL}(0.647 \pm 0.046$ and $0.700 \pm 0.110$, respectively). Higher LPS concentrations led to a mean decrease in mean nuclear p65 density, potentially indicating that the higher doses may be cytotoxic (Figure 2). The coefficient of variation values for the experiments was between $7 \%$ and $28.7 \%$ for individual doses.

\section{THP-I monocytes showed a greater overall increase in nuclear p65 compared to RAW 264.7 macrophages}

THP-1 monocytes did not only respond to lower doses such as $0.1 \mathrm{ng} / \mathrm{mL}$ but also showed a greater increase in nuclear p65. Similar numbers of cells were used in each experiment (THP-1 cells: $5 \times 10^{6}$ in $7 \mathrm{~mL}$ of media and RAW cells: $5 \times 10^{6}$ in $5 \mathrm{~mL}$ of media). See Figure 2 for a comparison of p65:actin ratio at similar doses in both cell lines, the graph also demonstrates how RAW 264.7 macrophages required higher doses of LPS for p65 activation and significant elevations from basal levels.
Both THP-I and RAW 264.7 cells showed a dose-response relationship in activation of the NF- $\mathrm{KB}$ pathway in response to LPS

Comparison of the curves suggests that THP-1 cells respond to lower doses of LPS than RAW 264.7 cells and lower doses produce a greater fold increase in the nuclear p65 density, indicating that they are more sensitive to lower concentrations (Figure 3).

\section{IP stool inoculation leads to an acute suppurative peritonitis, which is not observed after IP LPS treatment}

Animals that had IP stool inoculation exhibited an acute suppurative perihepatitis, represented by a variably thick layer of NLs attached to the liver surface. These were mixed with amorphous granular eosinophilic material (fecal matter) and occasional bacterial colonies from the suspension. This was underlain by a thin sub-serosal layer of NLs (Figure 4B). This can be compared to the control animals who received IP saline (Figure 4A). In addition, a few to several NLs were found in the lumen of central veins (CVs) and portal veins. Large numbers of NLs in the blood vessels indicate a release of NLs from storage pools (ie, bone marrow) and a neutrophilia - a finding consistent with systemic inflammation. Rarely, individual NLs were also seen in the sinusoids immediately adjacent to the CVs. The PAS reaction highlighted diffuse hepatocellular glycogen accumulation, which was most pronounced in centrilobular areas. 


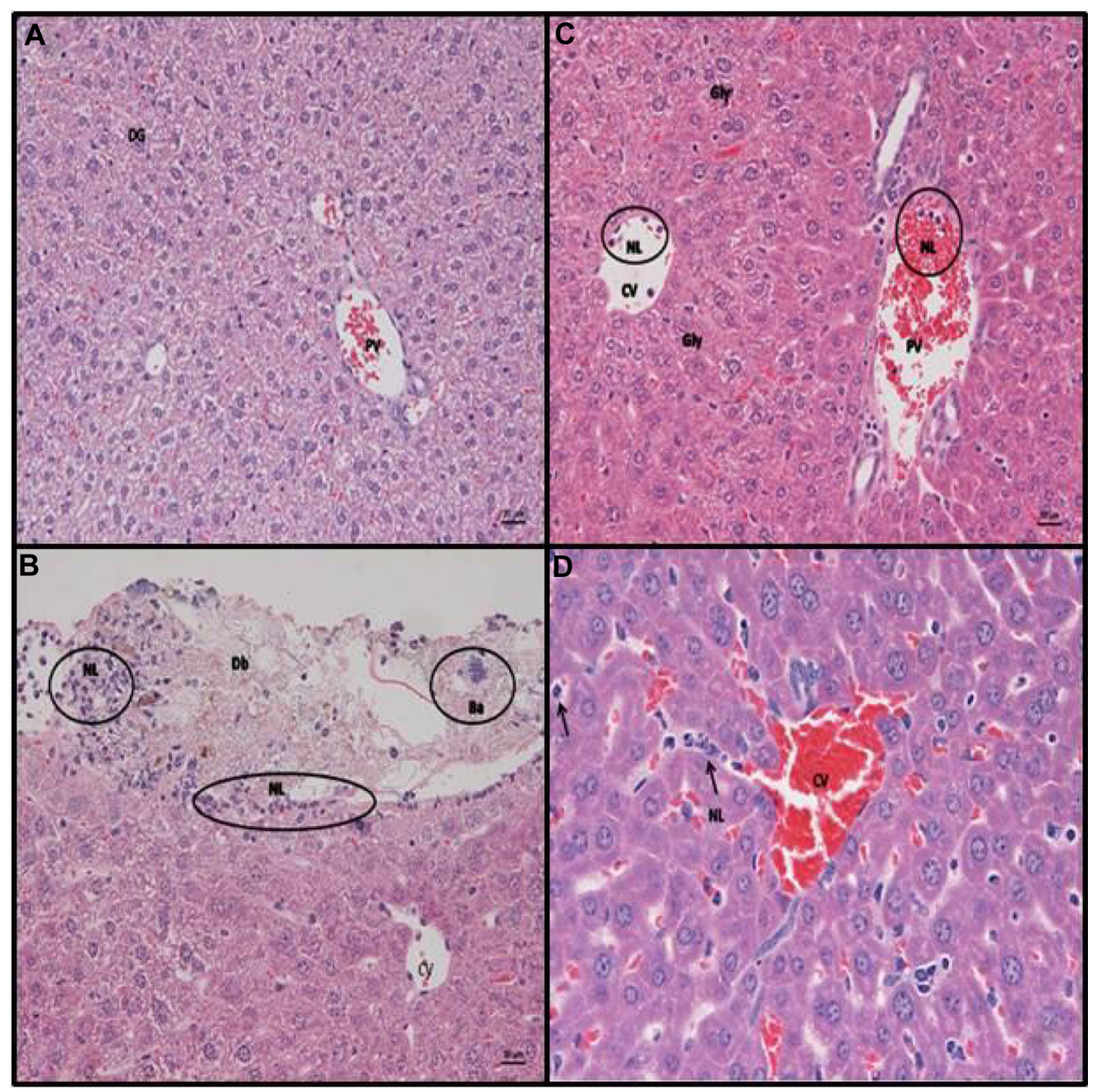

Figure 4 Representative slides from liver for animals receiving IP saline/LPS/stool suspension.

Notes: Hematoxylin and eosin staining of C57BL/6 mouse liver at 6 hours post (A) intraperitoneal (IP) injection with saline. Normal histology noted, DG (B) IP inoculation of stool ( $5 \mu \mathrm{L} / \mathrm{g}$ body weight ). Liver parenchyma with capsule, exhibiting focal serosal attachment of NLs and cellular debris (perihepatitis). Underlying the infiltrate is a thin layer of subserosal neutrophils. Ba from the stool suspension can also be seen. (C and D) IP injection with LPS (25 mg/kg). Liver parenchyma with neutrophils in the lumen of $\mathrm{CVs}$ and PVs. Closer view of the liver parenchyma highlights the increased amount of neutrophils between hepatic cords. Hepatocytes do not exhibit cytoplasmic vacuolation, consistent with a diffuse loss of glycogen (scale bar $=20 \mu \mathrm{m}, \mathbf{A}-\mathbf{C}$; scale bar $=10 \mu \mathrm{m}, \mathbf{D}$ ). Representative examples of neutrophils are circled "NL" in B and C and arrowed "NL" rolling along the hepatic chords in $\mathbf{D}$. Bacterial colonies are circled "Ba" in $\mathbf{B}$.

Abbreviations: Db, bacterial debris; Gly, glycogen; LPS, lipopolysaccharide; NLs, neurtrophils; CVs, central veins; PVs, portal veins; Ba, bacterial colonies; DG, diffuse glycogen.

\section{IP LPS treatment induces more} pronounced systemic NL recruitment into the liver than IP stool inoculation

\section{at 6 hours}

LPS-treated animals did not show any evidence of perihepatitis. However, they exhibited more intense leukocyte recruitment into the parenchyma, as represented by the presence of increased numbers of disseminated NLs between hepatic cords. NLs were also present in the lumen of CVs and portal veins, in similar amounts to those observed in the mice that had undergone IP stool inoculation. In addition, diffuse glycogen loss was observed. This was represented by a loss of hepatocellular cytoplasmic vacuolation and the absence of PAS-positive material within hepatocytes (see Figure 4C and D).

\section{IP stool inoculation results in more substantial NL recruitment into tissues such as fat and skeletal muscle than IP LPS treatment}

Mice treated with LPS and stool inoculation showed variable evidence of NL recruitment, represented by the presence of veins in which NLs were found in the lumen and rolling along (attached to) endothelial cells (Figure 5). This was more frequent in mice that had been inoculated with the stool suspension, indicating an early onset of systemic NL recruitment into these tissues in these animals. In IP LPS-treated animals, the skeletal muscle (gastrocnemius) did not exhibit any pathological changes. Mice that had been inoculated with stool suspension often exhibited variable amounts of NLs in the lumen of veins (Figure 5). 


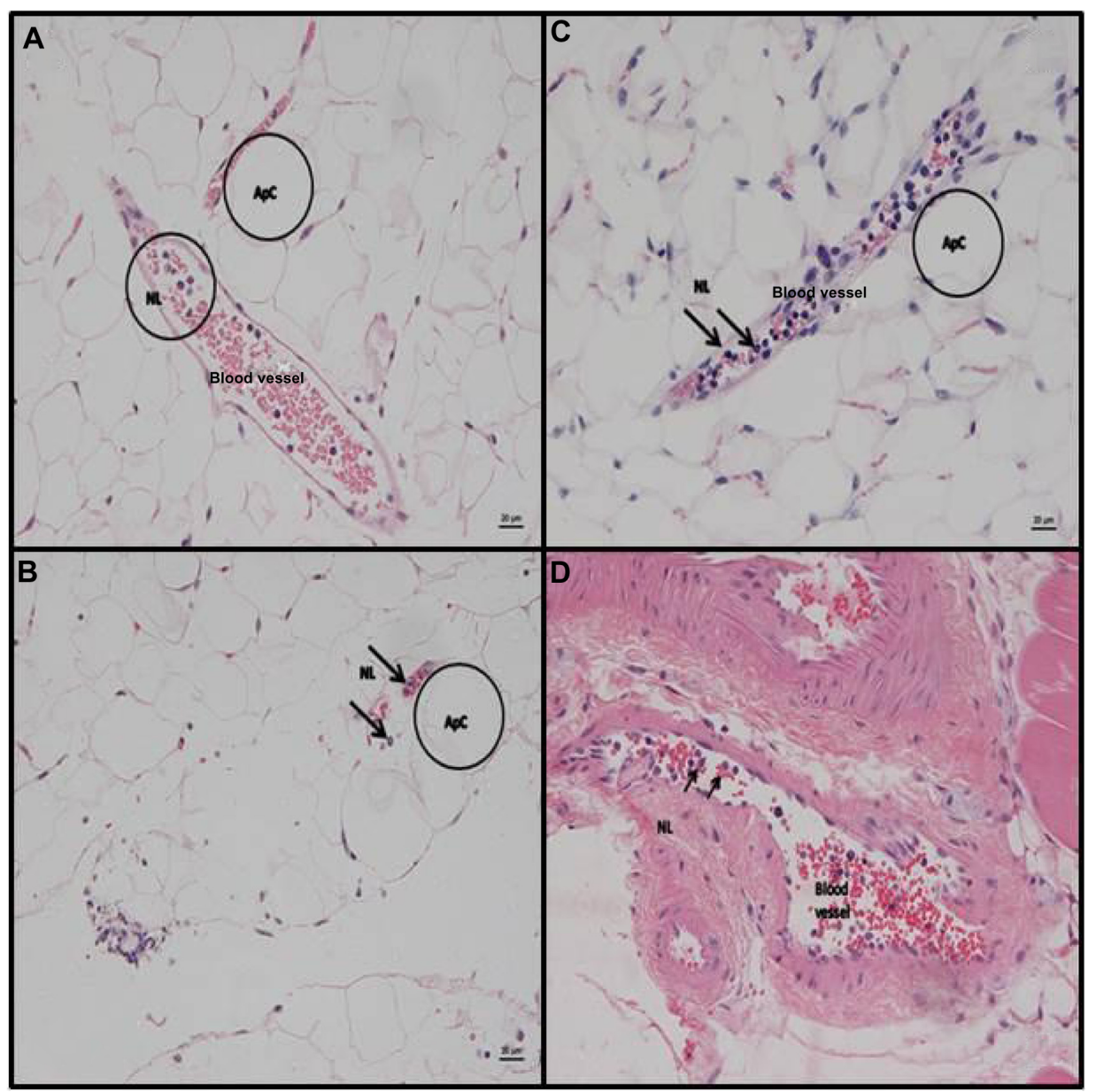

Figure 5 Representative slides from adipose tissue and muscle for animals receiving IP stool suspension.

Notes: Hematoxylin and eosin staining (H\&E) of C57BL/6 mouse adipose tissue and muscle at 6 hours post- IP inoculation of stool (5 $\mu$ L/g body weight ). (A) Epididymal adipose tissue. Embedded between ApCs are veins that exhibit several neutrophils in the lumen. (B) Peri-renal fat with focal serosal neutrophil infiltration with mixed bacteria (peritonitis) and veins containing neutrophils in the lumen. (C) Epididymal adipose tissue. Vein packed with NLs. H\&E stain. (D) Muscle gastrocnemius. Larger artery with several neutrophils in the lumen (scale bar $=20 \mu \mathrm{m}, \mathbf{A}-\mathbf{D}$ ). Representative adipocytes ApC are circled "Apc" in A, B, and C. A circle labeled "NL" marks a representative group of neutrophils in $\mathbf{A}$ and individual rolling neutrophils are arrowed "NL" in $\mathbf{D}$.

Abbreviations: IP, intraperitoneal; LPS, lipopolysaccharide; ApCs, adipocytes; NLs, neutrophils.

\section{Discussion}

\section{Differences in cellular response to LPS}

Our experiments demonstrate that p65 detection through immunoblotting is a valuable, reliable way of detecting activation of the NF- $\kappa B$ pathway in cells dosed with LPS. Gradual increased dosing of LPS resulted in a greater translocation of the $\mathrm{p} 65$ subunit of NF- $\mathrm{KB}$ from the cytoplasm to the nucleus of the cell, in both human and murine cells. However, it appeared that the murine cell line required a higher dose of LPS than human cells, indicating resilience to endotoxin.

The different dose-response to LPS in human and murine cells has been reported earlier, with RAW 264.7 cells requiring higher doses of LPS in order to elicit similar responses to those seen in THP1 cells. ${ }^{12}$ TLR4 is an important receptor expressed on most myeloid cell derivatives that allows them to detect and respond to LPS. ${ }^{13}$ In vitro studies have shown that human macrophages up-regulate and increase their activation of TLR4 on stimulation with LPS, whereas murine cells appear to down-regulate TLR4 transcripts under similar conditions. ${ }^{14}$ This fundamental variation in the expression pattern could explain the higher levels of p65 translocation in human but not murine cells seen in our experiments.

Lymphocyte antigen 6 (or MD-2), an extracellular protein and ligand of the TLR4, is important in LPS binding to this receptor. ${ }^{15}$ Hajjar et al ${ }^{12}$ demonstrated that the human TLR4-MD-2 complex is more sensitive in humans than in mice and has the ability to recognize acetylation of the LPS molecule allowing it to generate a more specific inflammatory response in humans. Warren et $\mathrm{al}^{16}$ suggested that the differences in resilience to LPS between species, with humans toward one end and rodents toward the other end of a spectrum, may not be due to an intrinsic cellular process, but rather may be related to mechanisms involving and perhaps regulated 
by serum proteins. This was demonstrated by the ability of isolated mouse serum to suppress the LPS-induced TNF $\alpha$ production in macrophages to a greater extent than human serum. Although our experiments did not explore the molecular mechanisms leading to NF- $\mathrm{\kappa B}$ activation, our results support the observation that human cells are more responsive to LPS treatment than murine cell lines. Hence, the suitability of murine animal models to mimic NF- $\kappa \mathrm{B}$ activation as a key pathway in human sepsis needs to be questioned.

\section{Differences in monocyte and macrophage response to LPS}

Upon differentiation to macrophages, monocytes lose their ability to multiply and their immune functions and antibacterial properties become enhanced. The monocyte cell itself has been shown to be less sensitive to LPS than the macrophage in vitro and this was shown by accumulation of NF- $\kappa B$ in the cytoplasm of differentiated THP-1 cells in comparison to non-differentiated cells. ${ }^{17}$ Takashiba et al ${ }^{17}$ concluded that this accumulation was a priming event in making the macrophage more sensitive to LPS stimulation and resulted in a more rapid NF- $\mathrm{NB}$ translocation and overall higher nuclear content. Therefore, higher levels of $\mathrm{p} 65$ translocation in a primed highly sensitive macrophage compared to a monocyte would have been expected. The results within this study demonstrated less nuclear translocation of p65 in the murine macrophage in comparison to the human monocyte; these results further highlight the role of species variation ${ }^{12,17}$ to LPS.

\section{IP LPS versus IP stool inoculation model of sepsis}

IP-dosed LPS mice showed no evidence of peritonitis, whereas IP stool inoculation resulted in an acute suppurative peritonitis induced by the bacteria from the inoculum. This shows that the latter model reflects the pathogenesis of bacterial sepsis of any origin (in line with ACCP/SCCM definitions ${ }^{4}$ ) much better than the LPS model. The LPS model should be considered as a model of "sterile inflammation", resulting in substantial generalized endotoxemia.

\section{Comparison of NL recruitment in both models}

In response to bacterial infection, recruitment and tissue infiltration of NLs are hallmarks of early immune activation. ${ }^{18}$ However, rapid cessation of NL emigration into inflamed tissue is required to initiate resolution of infection.

Both models (LPS and stool inoculation) investigated in this study showed principal changes consistent with NL recruitment into tissues; IP LPS animals had a tendency to show greater numbers of NLs than the IP stool-inoculated animals, an observation which could be related to the higher concentrations of cytokines that are seen with the LPS model. IP stool-inoculated animals demonstrated occasional recruitment of NLs into adipose tissue and skeletal muscle which was absent in IP LPS animals. This reflects the diffuse systemic recruitment seen in human sepsis and highlights the superiority of this model in recapitulating a human sepsis environment in vivo.

The recruitment and infiltration of NLs into the tissues were determined at 6 hours in both models to reflect the acute nature of the inflammatory response. Whether longer stimulation periods lead to more pronounced inflammatory changes, including persistent NL recruitment, have to be investigated in future studies.

Our results demonstrated that mice dosed IP with stool suspension had more numbers of NLs in secondary tissues such as adipose tissue and skeletal muscle. Using gene knockout mice, Andonegui et al ${ }^{19}$ showed that endothelium-derived TLR4 was more important than leukocyte-derived TLR4 in LPS-induced NL sequestration into the lungs. Considering the histopathological results of our experiments, the key difference between both models could lie in the level, extent, and tissue pattern of endothelial activation, where in contrast to the IP LPS model, the more gradual rise and the sustained levels of cytokines seen by Gonnert et $\mathrm{al}^{10}$, favors a wider, more diffuse systemic response and recruitment of leukocytes into tissues.

\section{Limitations}

Our study has several limitations in that we did not measure cytokine levels in blood or tissue after IP LPS challenge or IP stool inoculation. However, previous studies have thoroughly described the characteristics of cytokine release in both models. ${ }^{5,6,10}$ In general, the use of cell lines is limited by the fact that the lines themselves are cancerous and several mutations are required to render the cells immortal, a process that may alter the biological properties of the cells completely when compared to in vivo inflammation. We therefore used two different animal models (IP LPS and stool inoculation) to investigate whether both immune stimulants produce histopathological features comparable to those observed in human sepsis. To our knowledge, this is the first report demonstrating that stool inoculation compared to LPS induces changes consistent with acute suppurative peritonitis, although these signs were milder than found in severe human intra-abdominal sepsis. This highlights the importance of sequential experiments to establish a 
time course of the inflammatory response and its relation with histopathological changes. Furthermore, since other pathways such as those involving interferon regulatory transcription factors and mitogen-activated protein kinases can be activated through TLRs, contributing toward cytokine production and inflammation, any experiments in the future should aim to characterize their role.

\section{Conclusion}

Our results demonstrate the following: Immunoblotting p65 is a reliable, detectable, and reproducible way of measuring dose-dependent translocation of p65 from the cytoplasm into the nucleus; Comparison of the human and murine cell lines showed significant, fundamental differences in p65 translocation between human and murine myeloid cell lines; Comparison between LPS-challenged and stool-inoculated mice demonstrated histopathological signs of acute suppurative peritonitis after stool inoculation, whereas LPS induced a "sterile inflammation".

In summary, our results support previous observations that translation of murine models into the human clinical setting suffers from considerable limitations in that speciesassociated differences in LPS response and the histopathological changes during clinical sepsis cannot be reproduced adequately by injection of LPS. Therefore, the so-called translational disconnect that exists between murine LPS models and human sepsis involves NF- $\kappa \mathrm{B}$ activation at a molecular level and is further augmented by the use of LPS as a stimulus for infectious responses in vivo.

\section{Author contributions}

All authors contributed toward data analysis, drafting and critically revising the paper and agree to be accountable for all aspects of the work.

\section{Disclosure}

The authors report no conflicts of interest in this work.

\section{References}

1. Bonizzi G, Karin M. The two NF-kappaB activation pathways and their role in innate and adaptive immunity. Trends Immunol. 2004; 25(6):2808.

Journal of Inflammation Research

\section{Publish your work in this journal}

The Journal of Inflammation Research is an international, peer-reviewed open-access journal that welcomes laboratory and clinical findings on the molecular basis, cell biology and pharmacology of inflammation including original research, reviews, symposium reports, hypothesis formation and commentaries on: acute/chronic inflammation; mediators of inflamma-
2. Sun Z, Andersson R. NF-kappaB activation and inhibition: a review. Shock. 2002;18(2):99-106.

3. May MJ, Ghosh S. Signal transduction through NF-kappa B. Immunol Today. 1998;19(2):80-88.

4. Bone RC, Balk RA, Cerra FB, et al. Definitions for sepsis and organ failure and guidelines for the use of innovative therapies in sepsis. The ACCP/SCCM Consensus Conference Committee. American College of Chest Physicians/Society of Critical Care Medicine. Chest. 1992;101(6): 1644-1655.

5. Rock KL, Latz E, Ontiveros F, Kono H. The sterile inflammatory response. Annu Rev Immunol. 2010;28:321-342.

6. Remick DG, Ward PA. Evaluation of endotoxin models for the study of sepsis. Shock. 2005;24(Suppl 1):7-11.

7. Dyson A, Singer M. Animal models of sepsis: why does preclinical efficacy fail to translate to the clinical setting? Critical Care Med.2009; 37(Suppl):S30-S37.

8. Doi K, Leelahavanichkul A, Yuen PS, Star RA. Animal models of sepsis and sepsis-induced kidney injury. J Clin Invest. 2009;119(10): $2868-2878$.

9. Reinhart K, Karzai W. Anti-tumor necrosis factor therapy in sepsis: update on clinical trials and lessons learned. Crit Care Med. 2001; 29(Suppl):S121-S125.

10. Gonnert FA, Recknagel P, Seidel M, et al. Characteristics of clinical sepsis reflected in a reliable and reproducible rodent sepsis model. J Surg Res. 2011;170(1):e123-e134.

11. Van Amersfoort ES, Van Berkel TJ, Kuiper J. Receptors, mediators, and mechanisms involved in bacterial sepsis and septic shock. Clin Microbiol Rev. 2003;16(3):379-414.

12. Hajjar AM, Ernst RK, Tsai JH, Wilson CB, Miller SI. Human Toll-like receptor 4 recognizes host-specific LPS modifications. Nat Immunol. 2002;(4):354-359.

13. Matsuguchi T, Musikacharoen T, Ogawa T, Yoshikai Y. Gene expressions of Toll-like receptor 2, but not Toll-like receptor 4, is induced by LPS and inflammatory cytokines in mouse macrophages. J Immunol. 2000;165(10):5767-5772.

14. Rehli M. Of mice and men: species variations of Toll-like receptor expression. Trends Immunol. 2002;23(8):375-378.

15. Visintin A, Mazzoni A, Spitzer JA, Segal DM. Secreted MD-2 is a large polymeric protein that efficiently confers lipopolysaccharide sensitivity to Toll-like receptor 4. Proc Natl Acad Sci U S A. 2001;98(21): 12156-12161.

16. Warren HS, Fitting C, Hoff E, et al. Resilience to bacterial infection: difference between species could be due to proteins in serum. $J$ Infect Dis. 2010;201(2):223-232.

17. Takashiba S, Van Dyke TE, Amar S, Murayama Y, Soskolne AW, Shapira L. Differentiation of monocytes to macrophages primes cells for lipopolysaccharide stimulation via accumulation of cytoplasmic nuclear factor kappaB. Infect Immun. 1999;67(11):5573-5578.

18. Park JS, Svetkauskaite D, He Q, et al. Involvement of toll-like receptors 2 and 4 in cellular activation by high mobility group box 1 protein. J Biol Chem. 2004;279(9):7370-7377.

19. Andonegui G, Bonder CS, Green F, et al. Endothelium-derived toll-like receptor-4 is the key molecule in LPS-induced neutrophil sequestration into lungs. J Clin Invest. 2003;111(7):1011-1020.

\section{Dovepress}

tion; cellular processes; molecular mechanisms; pharmacology and novel anti-inflammatory drugs; clinical conditions involving inflammation. The manuscript management system is completely online and includes a very quick and fair peer-review system. Visit http://www.dovepress.com/ testimonials.php to read real quotes from published authors. 